\title{
On the Total Vertex Irregular Labeling of Proper Interval Graphs
}

\author{
A. Rana* \\ Department of Mathematics, Narajole Raj College, Paschim Medinipur, West Bengal, 721211, India
}

Received 12 March 2020, accepted in final revised form 21 May 2020

\begin{abstract}
A labeling of a graph is a mapping that maps some set of graph elements to a set of numbers (usually positive integers). For a simple graph $\mathrm{G}=(\mathrm{V}, \mathrm{E})$ with vertex set $\mathrm{V}$ and edge set $\mathrm{E}$, a labeling $\Phi: \mathrm{V} \cup \mathrm{E} \rightarrow\{1,2, \ldots, k\}$ is called total $k$-labeling. The associated vertex weight of a vertex $\mathrm{x} \in \mathrm{V}$ under a total $k$-labeling $\Phi$ is defined as $\mathrm{wt}(x)=\Phi(x)+\sum_{\mathrm{y} \in \mathrm{N}(\mathrm{x})} \Phi(x y)$ where $\mathrm{N}(x)$ is the set of neighbors of the vertex $x$. A total $k$-labeling is defined to be a vertex irregular total labeling of a graph, if for every two different vertices $x$ and y of $G$, $\operatorname{wt}(x) \neq \operatorname{wt}(y)$. The minimum $k$ for which a graph $G$ has a vertex irregular total $k$-labeling is called the total vertex irregularity strength of $G, t v s(G)$. In this paper, total vertex irregularity strength of interval graphs is studied. In particular, an efficient algorithm is designed to compute $t v s$ of proper interval graphs and bounds of $t v s$ is presented for interval graphs.
\end{abstract}

Keywords: Design of algorithms; Interval graphs; Vertex irregular total labelling; Total vertex irregularity strength.

(C) 2020 JSR Publications. ISSN: 2070-0237 (Print); 2070-0245 (Online). All rights reserved. doi: http://dx.doi.org/10.3329/jsr.v12i4.45923

J. Sci. Res. 12 (4), 537-543 (2020)

\section{Introduction}

In graph theory, the field of graph labeling is growing fast during the last three decades. Labeled graphs has wide range of applications including coding theory, x-ray, crystallography, radar, astronomy, circuit design, channel assignments of FM radio stations and communication network addressing. A labeling of a graph is a function from the vertex set $V$ or the edge set $E$ or both to the set of natural numbers subject to certain conditions. If the domain is the vertex-set (edge-set) the labeling is called vertex-labeling (edge labeling). For a graph $G=(V, E)$ a labeling $: V \cup E \rightarrow\{1,2, \ldots, k\}$ is said to be a vertex irregular total $k$-labeling of the graph $G$ if for every two different vertices $x$ and $y$ of $G, w t(x) \neq w t(y)$ where the weight of a vertex $x$ in the labeling $\Phi$ is

$$
\operatorname{wt}(x)=\Phi(x)+\sum_{y \in \mathbb{N}(x)} \Phi(x y)
$$

where $N(x)$ is the set of neighbors of $x$. The minimum $k$ for which the graph $G$ has an vertex irregular total $k$-labeling is called the total vertex irregularity strength of the graph

\footnotetext{
* Corresponding author: arnrc79@gmail.com
} 
$\mathrm{G}$ and is denoted by $\operatorname{tvs}(G)$. The irregularity strength $s(G)$ of a graph $G$ can be defined only for graphs containing at most one isolated vertex and no connected component of order 2. On the other hand, the total vertex irregularity strength $\operatorname{tvs}(G)$ is defined for every graph $G$. This paper dedicated to the study of total vertex irregularity strength of a subclass of interval graphs, called proper interval graphs.

An undirected graph $G=(V, E)$ is an interval graph, if the vertex set $V$ can be put into one to one correspondence with a set of intervals $I$ on the real line $R$ such that two vertices are adjacent in $G$, if and only if their corresponding intervals have non empty intersection. A graph $G$ is a proper interval graph, if there is an interval representation of $G$ in which no interval properly contains another.
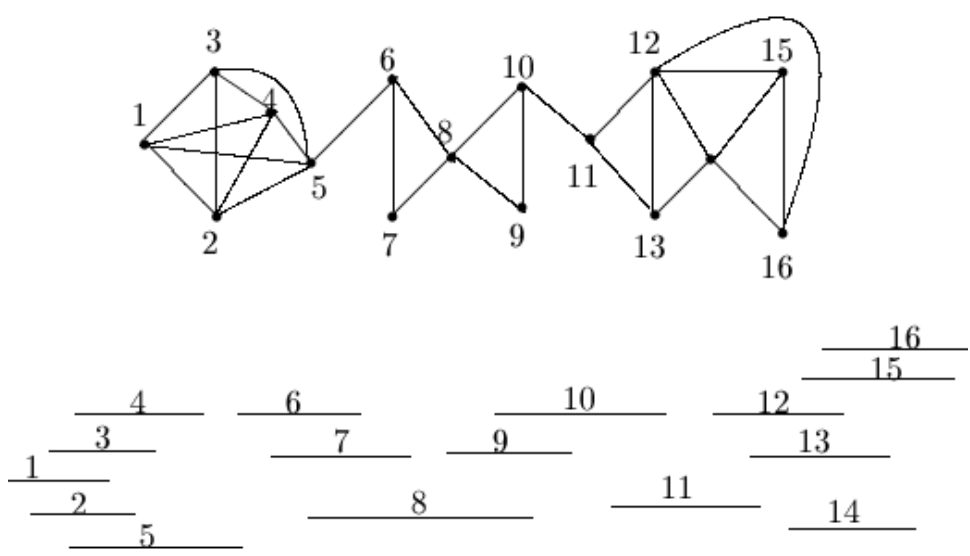

Fig. 1. An Interval graph with its interval representation.

The intervals and the vertices of an interval graph are very similar things. Interval graphs are discussed extensively in [1]. Here, we assume that the input graph is given by an interval representation $I$ which is the set of $n$ sorted intervals labeled by $1,2, \ldots, n$.

Let $I=\left\{I_{1}, I_{2}, \ldots, I_{n}\right\}$ where $I_{j}=\left[a_{j}, b_{j}\right], j=1,2,3, \ldots, n$; be the interval representation of the given interval graph $G=(V, E), V=\{1,2, \ldots, n\}, a_{j}$ and $b_{j}$ are the left and the right end points of the interval $I_{j}$. Without any loss of generality, we assume that each interval contains both its end points and that no two intervals share a common end point. Also, we assume that the intervals in $I$ are indexed by increasing right end points, that is, $b_{1}<b_{2}<$. $\ldots<b_{n}$. This indexing is known as IG ordering. Fig. 1 shows an interval graph and its corresponding interval representation. An interval graph can be recognized in $O(n+m)$ time which yields an interval representation in the certain instance. All graphs considered in this paper are connected, undirected and have no self loop or parallel edges.

The study of graphs labeling was initiated by Sadlacek in 1964 [2]. This study was continued by Stewart [3]. In 1986 Chartrand et al. [4] introduced irregular assignments and the irregularity strength of graphs. The motivation of Bača et al. [5] behind the definition of total irregularity strength of graphs came from elsewhere [4]. A lot of work is done for both vertex and edge variants of total irregularity strength of graphs [5-9,10-15]. 
To date, the total vertex irregularity strengths are known only for some special classes of graphs. Bača et al. determined exact value of tvs for stars, cliques and prisms [5]. Also, they fixed boundary value of $t v s$ for tree and $(p, q)$ graph.

If $G$ is a $(p, q)$ graph with minimum degree $\delta$ and maximum degree $\Delta$, then Bača et al. [5] proved that

$\left\lceil\frac{p+\delta}{\Delta+1}\right\rceil \leq \operatorname{tvs}(G) \leq p+\delta-2 \Delta+1$

These results were then improved by Przybylo [14] for sparse graphs and for graphs with large minimum degree. Anholcer et al. established a new upper bound of the form [9] $\operatorname{tvs}(G) \leq 3 \frac{p}{\delta}+1$.

Among the others, Ahmad et al. [6] fixed the lower bound of tvs of any graph. Moreover, Ahmad et al. [7,8] found an exact value of the total vertex irregularity strength for Jahangir graphs, circulant graphs and wheel related graphs. Wijaya and Slamin determined the exact values of the total vertex irregularity strength of wheels, fans, suns and friendship graphs [10]. Furthermore, Wijaya et al. found the total vertex irregularity strength for complete bipartite graphs [11]. Recently, a restricted variation of total irregular labeling is appeared in [15].

In this study we find the TVS of proper interval graphs, a subclass of interval graphs and present an algorithm to determine the TVS of proper interval graphs. Moreover, the boundary values of tvs of proper interval graphs and interval graphs are presented.

\section{Notations and Preliminaries}

A clique of a graph is a set of vertices, such that, there exists an edge between every pair of vertices in the set.

Lemma 1. (Fulkerson and Gross [16]). A triangulated graph and so an interval graph with $n$ vertices has at most $n$ maximal cliques. The number of maximal cliques is $n$ if and only if the graph has no edges.

A connected interval graph with $n$ vertices has at most $n-1$ maximal cliques, when the graph is a path. Gilmore and Hoffman have shown that the maximal cliques of an interval graph $G$ can be linearly ordered such that for every vertex $x \in V$ the maximal cliques containing $x$ occur consecutively [17].

Theorem 1. Let $G$ be an undirected graph. The following statements are equivalent [17].

(i) $G$ is an interval graph.

(ii) $G$ does not contain chordless cycle with four or more vertices and its complement $\bar{G}$ is a comparability graph.

(iii) The maximal cliques of $G$ can be linearly ordered such that for every vertex $x$ of $G$, the maximal cliques containing $x$ occur consecutively. 
A connected interval graph with $n$ vertices has at least 1 maximal clique and at most $n^{-1}$ maximal cliques. If the graph is a path then the number of maximal cliques is $n-1$ whereas it becomes a complete graph if the number of maximal clique is 1 . A clique with $n$ vertices is denoted by $C_{n}$.

Lemma 2. For a clique $C_{n}, t v s\left(C_{n}\right)=2$.

Proof: Let $C_{n}$ be a clique with $n$ vertices $v_{1}, v_{2}, \ldots, v_{n}$. Then there exists an edge between every pair of vertices $v_{i}$ and $v_{j}$. The edge between $v_{i}$ and $v_{j}$ is denoted by $e_{i j}$. We define a function $\phi: V \cup E \rightarrow\{1,2\}$ as follows:

$\phi\left(v_{1}\right)=1, \phi\left(e_{1 j}\right)=1, j=2,3, \ldots, n-1$ and $\phi\left(e_{1 n}\right)=1$.

$\phi\left(v_{2}\right)=2, \phi\left(e_{2 j}\right)=1, j=1,3,4, \ldots, n-1, \phi\left(e_{2 n}\right)=2$.

$\phi\left(v_{3}\right)=2, \phi\left(e_{3 j}\right)=1, j=1,2,4, \ldots, n-2, \phi\left(e_{3(n-1)}\right)=\phi\left(e_{3 n}\right)=2$.

$\ldots \ldots \ldots$

$\phi\left(v_{n}\right)=2, \phi\left(e_{n j}\right)=2, j=1,2, \ldots, n-1$.

Then weight of the vertices $v_{1}, v_{2}, \ldots, v_{n}$ are $n+1, n+2, \ldots, 2 n$ respectively. Therefore $\phi$ is a vertex irregular total labeling function and $C_{n}$ has vertex irregular total labeling. Clearly, 2 is the minimum integer such that $\phi: V \cup E \rightarrow\{1,2\}$ is a vertex irregular total labeling function.

Therefore

$\operatorname{tvs}\left(C_{n}\right)=2$.

The degree of all vertices of an interval graph $G$ can be obtained in $O\left(n^{2}\right)$ time. Let $D_{r}$ be the set of all vertices of $G$ of degree $r$. Then the vertices of $G$ can be partitioned into the sets $D_{\delta}, D_{\delta+1}, \ldots, D_{\Delta}$, where $\delta$ and $\Delta$ are the minimum and maximum degree of $G$ respectively.

Now, we prove a property of proper interval graph which plays an important role in our algorithm.

Lemma 3. The number of leaf vertex of a connected proper interval graph is at most 2.

Proof: Let $G$ be a connected proper interval graph with $n$ vertices $1,2, \ldots, n$. Therefore none of the intervals properly contain any other interval. Then the intervals $2,3, \ldots, n-$ 1 must intersect at least two intervals. This implies that the degree of each of the internal vertices $2,3, \ldots, n-1$ must be greater than 1 . Therefore, only the end vertices 1 and $n$ are potential leaf vertex.

From this lemma, it is observed that the cardinality of the set $D_{1}$ is 0 or 1 or 2 , that is, $\left|D_{1}\right| \leq 2$.

Lemma 4. For any graph $G$ with at least two vertices, $t v s(G) \geq 2$.

Proof: Let $G$ be graph with two vertices. Then degree of each vertex must be 1 . With the integer 1, we can label only one vertex and the weight of that vertex will be 2 (both vertex and edge get the label 1). To label the other vertex, another integer is needed except 1 . Therefore, at least two integers are required to label all the vertices of $G$.

Observe that, if it is possible to label the vertices and edges of a graph such that the weight of the vertices are consecutive integers starting from least possible weight, then $t v s$ is achieved. Our aim is to label the vertices and edges of $G$ in such a way that weight of 
the vertices are consecutive integers subject to the condition that the highest possible weight is least.

Lemma 5. If $\left|D_{k}\right|=(k+1) p+1(k>1)$ for some positive integer $p$, then at least $p+1$ integers are required to label the vertices of $D_{k}$ to follow vertex irregular total labeling.

Proof: Using the integer 1, we can label only one vertex of $D_{k}$. Each of the $k$ edges incident on the vertex receive the label 1 and the vertex also gets label 1 . Hence the weight of that vertex is $k+1$.

Using the integers 1 and 2, we can label $k+1$ vertices and edges incident on that vertices as $1,1,1, \ldots, 1,1$ ( $k+1$ terms) $; 1,1,1, \ldots, 1,2(k+1$ terms $) ; \ldots . . \quad \ldots . . ; 2,2,2, \ldots$, 2, 2 ( $k+1$ terms).

Hence by using the integers 1 and 2 , total $(k+1)+1$ vertices can be labeled.

Again, by using the integers 1,2 and 3, we can label $k+1$ vertices of $D_{k}$ and edges incident on that vertices as $2,2,2, \ldots, 2,2$ ( $k+1$ terms); $2,2,2, \ldots, 2,3$ ( $k+1$ terms); .... $\ldots ; 3,3,3, \ldots, 3,3(k+1$ terms). Hence by the integers 1,2 and 3 , total $2(k+1)+1$ vertices can be labeled. Proceeding with similar logic, $p(k+1)+1$ vertices of $D_{k}$ can be labeled by using $p+1$ integers $1,2, \ldots, p+1$.

Lemma 6. Let $\left|D_{k}\right|=(k+1) p+1(k>1$ and $p$ be an integer $)$ and $p+1$ consecutive integers are used to label the vertices of $D_{k}$. Then $p+1$ vertices of $D_{k+1}$ can be labeled using these $p+1$ consecutive integers.

Proof: Using $p+1$ consecutive integers $\{1,2, \ldots, p+1\}$, one can generate a maximum weight $(k+2)(p+1)$ of the vertices of $D_{k+1}$. In that case each of the $k+1$ edges and the only vertex get the label $p+1$. Again by using these $p+1$ consecutive integers, a maximum weight $(k+1)(p+1)$ is possible for the vertices of $D_{k}$. Hence $(k+2)(p+1)-(k$ $+1)(p+1)=p+1$ consecutive weights can be generated by using the same $p+1$ consecutive integers. These $p+1$ weights $(k+1)(p+1)+1,(k+1)(p+1)+2 \ldots,(k+$ $1)(p+1)+(p+1)$ can be used to label $p+1$ vertices of $D_{k+1}$.

Lemma 7. Let $\left|D_{k}\right|=(k+1) p+1(k>1$ and $p$ be a fraction) and $[p]+1$ consecutive integers are used to label the vertices of $D_{k}$. Then $(k+2)\lceil p\rceil-(k+1) p+1$ vertices of $D_{k+1}$ can be labeled by using these $[p]+1$ consecutive integers.

Proof: Similar logic as in the previous Lemma.

From the above two lemmas it is observed that without increasing the value of $t v s$, we can label some extra vertices of higher degree. The number of extra vertices of $D_{k+1}$ can be labeled by the integers used to label the vertices of $D_{k}$ is denoted by $e_{k+1}$. The values of $e_{i}$, $(i=3,4, \ldots, \Delta)$ can be determined by using previous two lemmas.

\section{The Algorithm}

The main idea of the proposed algorithm is follows. The algorithm proceeds by labeling the vertices in increasing order of degree of the given graph. At first, the sets $D_{1}, D_{2}, \ldots$, $D_{\Delta}$ are computed. Depending on the cardinal number of these sets, $t v s(G)$ is determined based on Lemma 4.

A formal description of the algorithm is given below. 


\subsection{Algorithm tvs}

Input: A set of $n(>1)$ sorted intervals of a proper interval graph $G$.

Output: Total vertex irregularity strength of $G$, that is, $t v s(G)$.

Initialize $i=2$ and $T=0$.

Step 1: Compute the sets $D_{1}, D_{2}, \ldots, D_{\Delta}$.

Step 2: If $\left|D_{1}\right|=0$ then

$e_{2}=0$, go to next step else if $\left|D_{1}\right|=1$ then

$T=T+1, e_{2}=1$ and go to next step

else $T=T+2, e_{2}=3$ and go to next step

end if.

Step 3: If $\left|D_{i}\right|=0$ then

$i=i+1$

If $i>\Delta$ go to last step 4. else $\left|D_{i}\right|=\left|D_{i}\right|-e_{i}$

If $\left|D_{i}\right|>0$ express $\left|D_{i}\right|=(i+1) p+1$

If $T>1, T=T+\lceil p\rceil, i=i+1$, repeat step 3

else $T=T+\lceil p\rceil+1, i=i+1$, repeat step 3

end if

else $i=i+1$, repeat step 3 end if

end if.

Step 4: $\operatorname{tvs}(G)=T$

\subsection{End tvs}

The correctness of the algorithm follows from the previous lemmas.

Theorem 2. For a proper interval graph $G$ with $n(>1)$ vertices,

$2 \leq t v s(G) \leq\left\lceil\frac{n-6}{3}\right\rceil$.

Proof: Observe that, if $G$ be a complete graph of order $n$ then $\operatorname{tvs}(G)=2$ (Lemma 2). If $G$ be not complete then $t v s(G)>2$. Therefore, $t v s(G) \geq 2$.

Again, the tvs of a graph $G$ will be maximum, if $G$ consist of maximum number of vertices of less degree. Now, a connected proper interval graph with $n$ vertices can have at most 2 leaf vertices and $n-2$ vertices of degree 2 . In this case, $G$ becomes a path $P_{n}$ of $n$ vertices.

Therefore $\left|D_{1}\right|=2$ and $\left|D_{2}\right|=n-2$. The integers 1 and 2 are necessary to label the vertices of $D_{1}$. Using these two integers, we can label 3 vertices of $D_{2}$, that is, $e_{2}=3$.

Hence, we have

$\operatorname{tvs}(G) \leq\left\lceil\frac{\mathrm{n}-6}{3}\right\rceil$.

Note that, the algorithm tvs does not work for general interval graph as the Lemma 2 is not true for general interval graph. The maximum number of leaf vertex of an interval graph is $n-1$. So, the algorithm needs modification depending on the number of leaf vertex to compute $t v s$ of a general interval graph. 
Theorem 3. For a connected interval graph $G$ with $n(>1)$ vertices,

$2 \leq \operatorname{tvs}(G) \leq\left\lceil\frac{n}{2}\right\rceil$.

Proof: The lower bound follows from the previous theorem.

Since maximum value of $t v s$ can be attained when the graph has the maximum number of vertices of less degree. Therefore, an upper bound is attained if the graph has maximum number of leaf vertex. Now, a connected interval graph $G$ with $n$ vertices can have at most $n-1$ vertices of degree 1 (leaf vertex). In that case, $G$ becomes a star and $\operatorname{TVS}(G)=\left\lceil\frac{n}{2}\right\rceil$.

Therefore, $2 \leq \operatorname{tvs}(G) \leq\left\lceil\frac{n}{2}\right\rceil$.

\section{Conclusion}

In this paper, an algorithm is designed to compute the exact value of tvs of proper interval graphs. Based on this algorithm the boundary values of $t v s$ presented for proper interval graphs and interval graphs. Our approach can be generalized to determine the $t v s$ of other intersection graphs, namely permutation graphs, trapezoid graphs and circular arc graphs. Future study can be done to find the value of $t v s$ for these intersection graphs.

\section{References}

1. M. C. Golumbic, Algorithmic Graph Theory and Perfect Graphs (Academic Press, New York, 1980). https://doi.org/10.1016/B978-0-12-289260-8.50010-8

2. J. Sedlacek, Theory of Graphs and Its Applications - Proceed. of the Sympos. Smolenice, (1963), pp. 163-167.

3. B. M. Stewart, Canad. J. Math. 18 (1966).

4. G. Chartrand, M. S. Jacobson, J. Lehel, O. R. Oellermann, S. Ruiz, and F. Saba, Congr. Numer. 64, 187 (1988).

5. M. Baca, S. Jendroll, M. Miller, and J. Ryan, Discrete Math. 307, 11 (2007). https://doi.org/10.1016/j.disc.2005.11.075

6. A. Ahmad, E. T. Baskoro, and M. Imran, Discuss. Math. Graph Theory 32, 3 (2012). https://doi.org/10.7151/dmgt.1619

7. A. Ahmad and M. Baca, Ars Combin. 112, 129 (2013).

8. A. Ahmad, K. M. Awan, I. Javaid, and Slamin, Aust. J. Combin. 51, 147 (2011).

9. M. Anholcer, M. Kalkowski, and J. Przybylo, Discrete Math. 309, 21 (2009).

10. K. Wijaya and Slamin, J. Combin. Math. Combin. Comput. 65, 103 (2008).

11. K. Wijaya, Slamin, Surahmat, and S. Jendrol, J. Combin. Math. Combin. Comput. 55, 129 (2005).

12. P. Jeyanthi and A. Sudha, J. Algorithms Comput. 48, 1 (2016).

13. P. Jeyanthi and A. Sudha, Palestine J. Math. 7, 2 (2018).

14. J. Przybylo, SIAM J. Discrete Math. 23, 1 (2009). https://doi.org/10.1137/070707385

15. A. Rana, Malaya J. Matematik 8, 2 (2020). https://doi.org/10.26637/MJM0801/0007

16. D. R. Fulkerson and O. A. Gross, Pacific J. Math. 15, 835 (1965).

17. P. C. Gilmore and A. J. Hoffman, Canad. J. Math. 16, 539 (1964). 